\title{
McArdle's disease presenting with acute renal failure
}

\begin{abstract}
A B Maharaj, V B Patel
Dr Ajesh Maharaj is a consultant physician/consultant rheumatologist and Head of the Department of Internal Medicine, Prince Mshiyeni Memorial Hospital and School of Clinical Medicine, College of Health Sciences, Nelson R Mandela School of Medicine, University of KwaZulu-Natal, Durban, South Africa. He is also affiliated to the Division of Clinical Immunology and Rheumatology, Academic Medical Center/University of Amsterdam, Netherlands. His current research interests include the genetics of psoriatic arthritis. Dr Vinod Patel is a consultant neurologist in the Department of Neurology, Inkosi Albert Luthuli Central Hospital and Nelson R Mandela School of Medicine, University of KwaZulu-Natal, Durban, South Africa.
\end{abstract}

Corresponding author: A B Maharaj (maharaja30@ukzn.ac.za)

We report on an interesting patient who presented with acute renal failure from rhabdomyolysis and was found to have McArdle's disease on further investigation.

S Afr Med J 2016;106(5):469. DOI:10.7196/SAMJ.2016.v106i5.10348

We report on a 35-year-old woman who presented to the emergency unit of a regional hospital with nonspecific symptoms. She was found to have acute renal failure. On clinical examination there were no signs of proximal muscle weakness and she appeared to be euthyroid. She had no features of collagen vascular disease. As part of the work-up for the acute renal failure she was noted to have an elevated creatine kinase (CK) level of $16343 \mathrm{IU} / \mathrm{L}$ (reference range $26-192$ IU/L). Results of thyroid function tests were normal. Antinuclear antibodies were negative. At this stage she was thought to have acute renal failure secondary to rhabdomyolysis.

Going back in the patient's history, she had had an unexplained compartment syndrome approximately 12 years ago that required surgical intervention. She was not investigated further for a cause for the compartment syndrome and had remained well since then despite exercising regularly. Prior to her current presentation she had gone on a long distance cycling trip.

The patient was admitted to the intensive care unit (ICU) and commenced on haemodialysis. While in the ICU, on complete bed rest, her CK level returned to normal, and she was discharged without any further intervention.

Two weeks later the patient returned for follow-up. She was completely asymptomatic, but measurement of the CK showed a level of $14934 \mathrm{IU} / \mathrm{L}$. She was readmitted to hospital and an ischaemic forearm lactate test was done. This showed a flat response of serum lactic acid and a fourfold increase in serum ammonium, which supported a

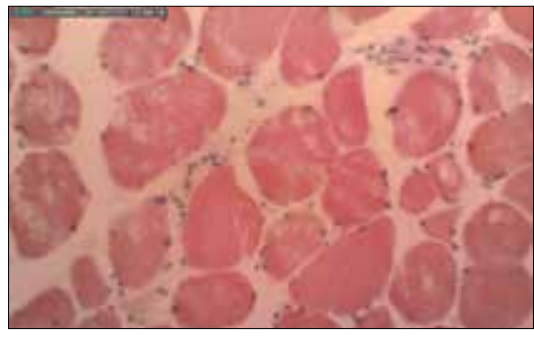

Fig. 1. Routine haematoxylin and eosin $(H \triangleleft E)$ stain shows mild variation in fibre size and shape.

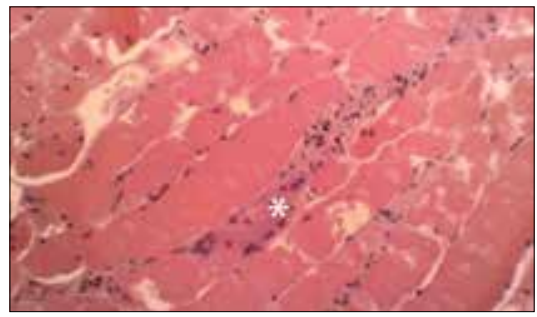

Fig. 2. Routine HeE stain shows a mild inflammatory reaction and degenerate/atrophic muscle fibres $(*)$.

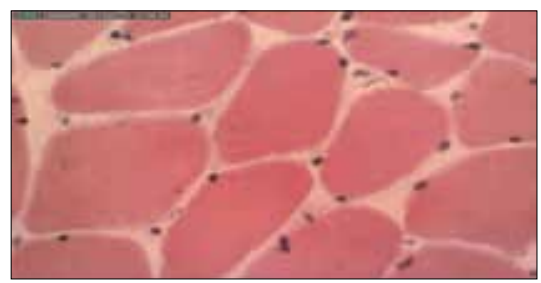

Fig. 3. Routine H\&E stain (normal control).

diagnosis of McArdle's disease. A muscle biopsy was then done (Figs 1 - 6).

The final diagnosis was McArdle's disease.

Accepted 2 December 2015.

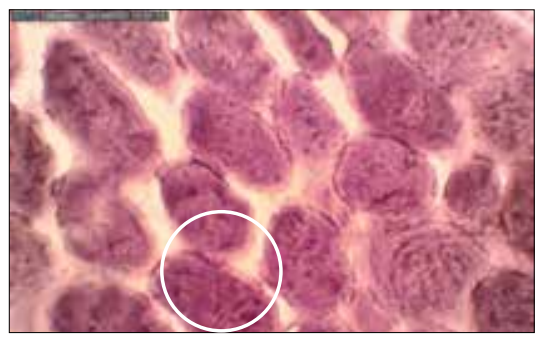

Fig. 4. The pink/eosinophilic stain confirms the presence of glycogen. There is focal subsarcolemmal accentuation of the glycogen (circled).

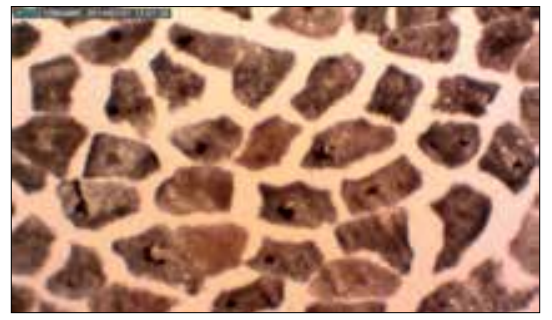

Fig. 5. Immunohistochemical staining in a control (normal) muscle biopsy specimen. The brown staining indicates the presence of myophosphorylase.

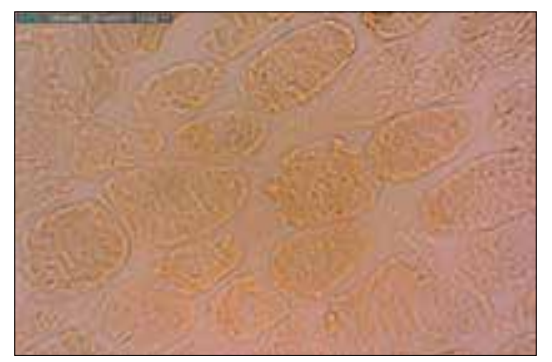

Fig. 6. Immunohistochemical staining in a muscle biopsy specimen from the patient. The lack of brown staining indicates the complete absence of myophosphorylase and is diagnostic of McArdle's disease. 